EPJ Web of Conferences 16, 03003 (2011)

DOI: $10.1051 /$ epjconf/20111603003

(C) Owned by the authors, published by EDP Sciences, 2011

\title{
Halftoning for high-contrast imaging
}

\author{
P. Martinez ${ }^{1, a}$, C. Dorrer ${ }^{2}$, E. Aller Carpentier ${ }^{1}$, M. Kasper $^{1}$, A. Boccaletti ${ }^{3}$ \\ and K. Dohlen ${ }^{4}$
}

\author{
${ }^{1}$ European Southern Observatory, Karl-Schwarzschild-Strasse 2, 85748 Garching, Germany \\ ${ }^{2}$ Aktiwave, 241 Ashley drive, Rochester, NY, 14620, USA; LESIA, Observatoire de Paris \\ Meudon, 5 pl. J. Janssen, 92195 Meudon, France \\ ${ }^{3}$ LAM, Laboratoire d'Astrophysique de Marseille, 38 rue Frédéric Joliot Curie, \\ 13388 Marseille Cedex 13, France
}

\begin{abstract}
High-contrast instruments, such as SPHERE (upcoming planet finder instrument for the ESO-VLT), or EPICS (planet hunter project for the future E-ELT), will require customized components with spatially varying transmission (e.g. coronagraphs, optical components that reduce the contrast between a companion and its parent star). The goal of these sub-systems is to control the spatial transmission, either in a pupil plane (pupil apodization), or in a focal plane of the instrument (occulting mask, i.e. lowfrequency filter). Reliably producing components with spatially varying transmission is not trivial, and different techniques have been already investigated for application to astronomy (e.g. metal deposition with spatially-varying thickness, or high-energy beam sensitive glass using e-beam lithography). We present some results related to the recent development of components with spatially varying transmission using a relatively simple technique analogous to the digital halftoning process used for printing applications.
\end{abstract}

\section{HALFTONE DOT PROCESS PRINCIPLE}

Halftoning has been used for hundreds of years in the printing industry as a solution for generating continuous-tone images with only black or white dots. The so-called halftone image, seen from a distance, should resemble the original continuous-tone image as much as possible, based on human vision perception. Following this idea, the continuous transmission of an optical filter can be generated with a specific implementation of opaque and transparent pixels as presented in Figure 1.

The resulting microdot filter is an array of dots (i.e. pixels) that are either opaque ( $0 \%$ transmission) or transparent $(100 \%$ transmission). It is fabricated by lithography of a light-blocking metal layer deposited on a transparent glass substrate. The technique has several advantages: relative ease of manufacturing, achromaticity, reproducibility, and ability to generate continuous and rapidly varying transmission functions, without introducing wavefront errors.

Several application-dependent parameters must be carefully defined. Microdot filters are arrays of pixels with binary transmission, and light propagating through them has a continuously varying intensity after free-space propagation, or after filtering in the far field. Therefore, these filters have a power spectrum different from the power spectrum of an ideal continuous filter. The way dots are distributed, the size of the dots, and the density of the dots in the pattern directly impact the power spectrum of the filter, and these parameters must be carefully studied accordingly with the application. Several studies have been carried out to quantify diffraction effects induced by the pixellation and binary transmission of the pixels. Diffraction stray-light analysis and guidelines for the design of pupilapodized coronagraphs (e.g. apodized pupil Lyot coronagraph, dual zone coronagraph, conventional pupil-apodization concepts) are available in $[1,2]$, while the specific case of focal-plane mask with

\footnotetext{
${ }^{\mathrm{a}}$ e-mail: martinez@eso.org
}

This is an Open Access article distributed under the terms of the Creative Commons Attribution-Noncommercial License 3.0, which permits unrestricted use, distribution, and reproduction in any noncommercial medium, provided the original work is properly cited. 


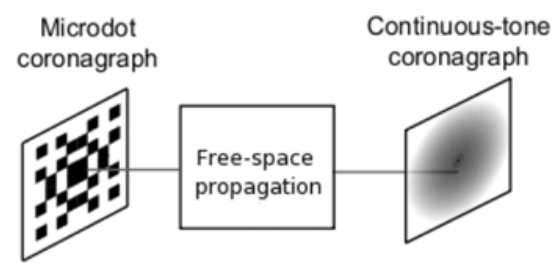

Figure 1. Principle of the generation of a continuous modified intensity transmission with a microdot filter.
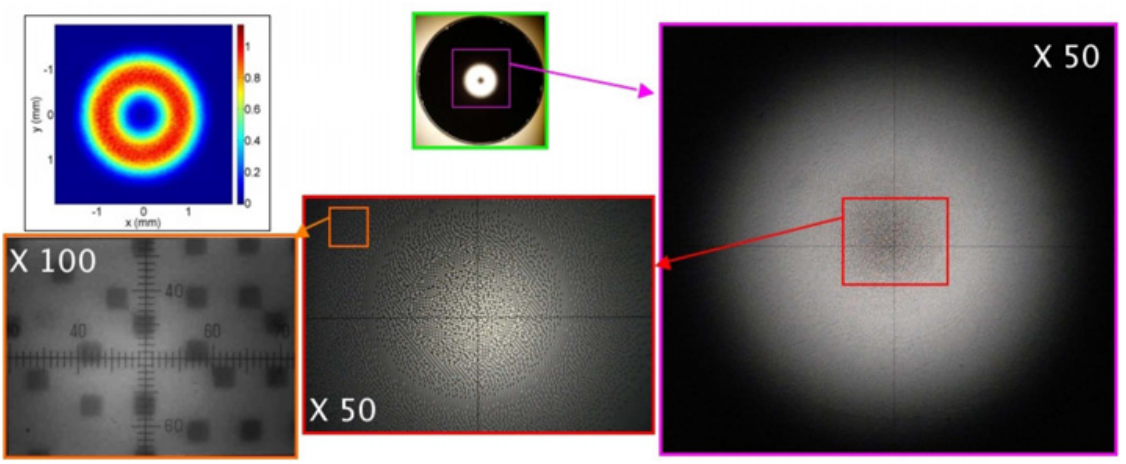

Figure 2. Metrology inspection of the APLC apodizer using a camera (green box), a shadowgraph (purple and red boxes), a microscope (orange box), and the corresponding spatially resolved transmission (top-left).

varying transmission (e.g. band-limited concepts) is presented in [3]. An overview of the technique for coronagraphy is also available in [4].

\section{DEVELOPMENTS FOR SPHERE AND EPICS CORONAGRAPHS}

The apodized pupil Lyot coronagraph (APLC) is one of the three coronagraphic concepts for the SPHERE (the Spectro-Polarimetric High-Contrast Exoplanet Research) near-IR instrument (IRDIS), which will enable direct images, and spectra for warm self-luminous Jovian planets. The APLC combines an apodizer in the entrance aperture with a small opaque mask in the focal plane. Manufacturing an accurate apodizer is critical for such coronagraph as for any other concept using pupil apodization. Although, a recent study also points out its potential application to ELT instruments [5] (e.g. EPICS, the Exo-Planet Imaging Camera and Spectrograph), besides the APLC, the Band-Limited coronagraph (BLC) is also considered and under investigations. This concept requires an accurate filter with a spatially varying transmission (e.g. a sinusoïdal function such as 1-sinc) in the focal plane of the instrument.

The APLC (4.5 $\lambda / \mathrm{D}$ configuration) apodizer is fabricated with $4.5 \times 4.5 \mu \mathrm{m}$ Chrome dots with regular optical density (4+ in the visible) on a $3 \mathrm{~mm}$ pupil size. Figure 2 illustrate the metrology inspection of the prototype. The profile transmission error has been evaluated to $3 \%$, while the achromaticity in transmission has been confirmed $(2 \%$ increase from a narrow $\mathrm{H}$-band filter to a broadband J-filter, Figure 4).

For the BLC, we have produced several prototypes (Figure 3) based on the same function (1-sinc), but with different dot sizes (indicated as a and $\mathrm{b}$ in Figure 3, where a corresponds to a $5 \mu \mathrm{m}$ dot size and $\mathrm{b}$ to a $10 \mu \mathrm{m}$ dot size) and function bandwidth (BL5 and BL10, which rules the inner working angle of the coronagraph). All prototypes have been manufactured with an Aluminium metal layer having a high optical density $(+8$ in the near-infrared) on a $12 \mathrm{~mm}$ diameter pattern. The high optical density specification is required for focal plane masks, whether they are fabricated with microdot filter or other 

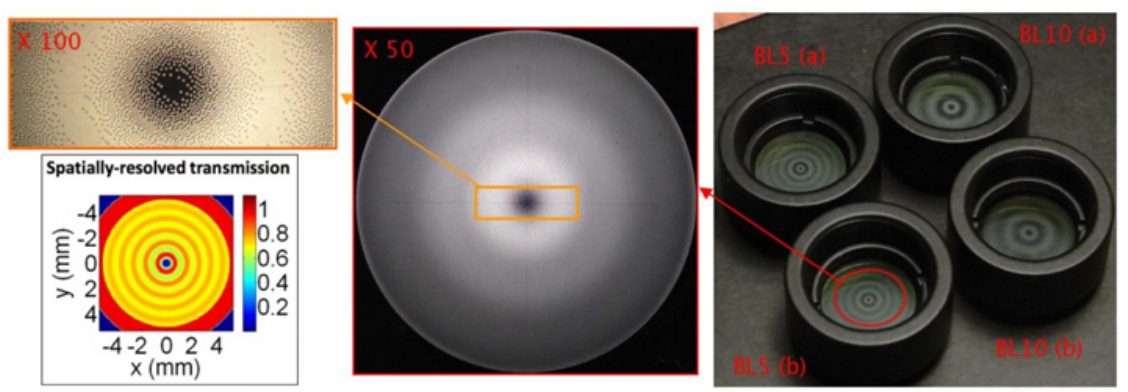

Figure 3. Metrology inspection of the final band-limited prototypes. From right to left: the four prototypes in their integration mounts, shadowgraph images $(\times 50$ and $\times 100)$, and spatially resolved transmission.
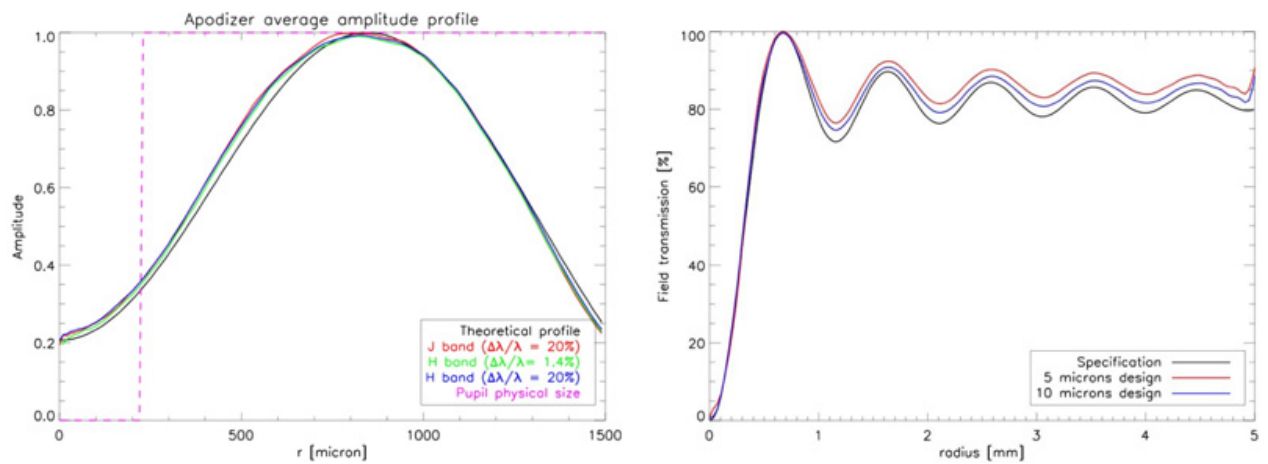

Figure 4. Apodizer azimuthally averaged measured profile (from the center to the edge) compared to specification using several filters (left), and similarly for the BL5 with 5 and $10 \mu \mathrm{m}$ dot sizes.

technologies, to prevent from starlight leaking on to the detector. Metrology inspection of the prototypes is illustrated in Figure 3. The accuracy has been measured to be of about 5\% in the high transmission part of the profile (improved to $2 \%$ with recent prototypes), while the low-transmission part is extremely good (Figure 4).

\section{PERFORMANCE OBTAINED WITH THE APLC AND BLCS IN THE NEAR-IR}

The prototypes have been tested in a near-infrared coronagraphic transmission bench developed at ESO (turbulence-free and AO-free, operating in H-band). The Strehl ratio of the bench has been evaluated to 94\%. In such conditions and assuming a VLT-like entrance pupil, the APLC demonstrates the ability to reduce the on-axis starlight by a factor of 700 on the peak, and to reach a contrast of $510^{-5}$ and $10^{-6}$ at 3 and $20 \lambda / \mathrm{D}\left(0.12\right.$ and $0.8^{\prime \prime}$ for a $8-\mathrm{m}$ telescope) respectively (see Figure 5). More détails can be found in [2].

BL5 demonstrates a peak reduction of 2500 , and a contrast of $310^{-5}$ and $2.710^{-8}$ at 5 (innerworking angle) and $20 \lambda / \mathrm{D}$ respectively (i.e. 0.2 and $0.8^{\prime \prime}$ ), while BL10 yields a peak reduction of 83000 and a contrast of $1.310^{-7}$ and $1.310^{-8}$ at 10 (inner-working angle) and $20 \lambda / \mathrm{D}$ respectively (see Figure 6). More détails can be found in [3].

The theoretical limits of the coronagraph have not been reached. Limitations originate from alignment issues, and from the static aberrations of the optical bench. Contrast levels reached are similar (APLC) or better (BLC) than the quasi-static speckle halo level expected after Adaptive Optics correction in a real instrument $\left(10^{-5}\right.$ to $\left.10^{-6}\right)$. 


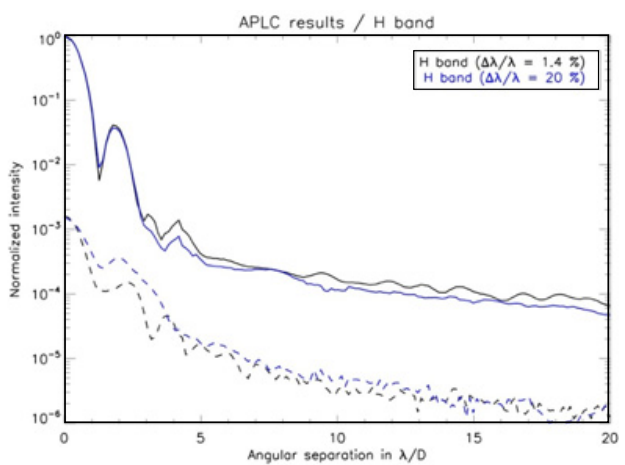

Figure 5. APLC results obtained in H-band assuming a VLT-like entrance pupil.
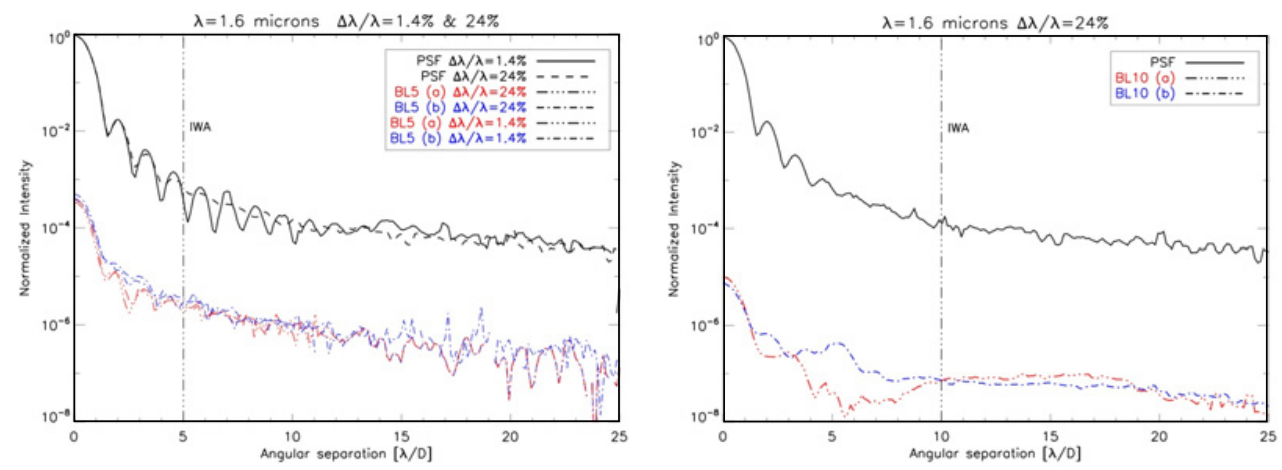

Figure 6. BL5 (left) and BL10 (right) results in H-band for different band pass filters assuming a fulfilled entrance pupil.

\section{CONCLUSION}

The halftoning process presented in this paper has been validated for pupil apodizer (APLC for SPHERE and GPI, Gemini Planet Imager), and is defined as the baseline technique for EPICS (for producing CPA - conventional pupil apodization coronagraph concept base-line). Space-based telescopes can also benefit from the halftoning process, such as the JWST NIRCam coronagraphs for which microdot bandlimited prototypes are being manufactured. It is important to note that the technique can be applied in many areas, such as laser beam shaping [6], intensity modulation in projection and illumination systems, or optical testing. Its interest has been recently extended to the manufacture of the apodization mask for the BIGRE Integral Field Spectrograph for SPHERE [7].

\section{References}

[1] P. Martinez, C. Dorrer, E. Aller Carpentier et al., A\&A. 495, 363-370 (2009)

[2] P. Martinez, C. Dorrer, M. Kasper et al., A\&A. 500, 1281-1285 (2009)

[3] P. Martinez, C. Dorrer, M. Kasper, ApJ, 705, 1637-1645 (2009)

[4] P. Martinez, C. Dorrer, E. Aller Carpentier et al., The Messenger, 137, 18-23 (2009)

[5] P. Martinez, A. Boccaletti, M. Kasper et al., A\&A. 492, 289-300 (2008)

[6] C. Dorrer, J. D. Zuegel, Opt. Soc. Am. B. 24, 1268 (2007)

[7] J. Antichi, K. Dohlen, R. Gratton et al., ApJ, 695, 1042-1057 (2009) 